\title{
Minimally Invasive Lateral Approach to the Thoracic Spine - Case Report and Literature Overview
}

Luiz Pimenta ${ }^{1}$, Luis Marchi ${ }^{2}$, Fernanda Fortti ${ }^{2}$, Leonardo Oliveira ${ }^{1 *}$, Rodrigo Amaral ${ }^{1}$ and Rubens Jensen ${ }^{1}$

${ }^{1}$ Instituto of Spine Pathology, Brazil

${ }^{2}$ Federal University of Sao Paulo, Sao Paulo, State of Sao Paulo, Brazil

\begin{abstract}
Background context: Alternatives to access the anterior portion of the thoracic spine include thoracotomy, thoracoscopy, and transpedicular approaches. These techniques have proven to be either extremely traumatic or to be technically difficult. Consequently, these approaches are not routinely used. The development of new monitoring systems, instruments, and implants allow conventional anterior thoracic surgery to be accomplished through a minimally disruptive way. The extreme lateral interbody fusion (XLIF) is one of these techniques.
\end{abstract}

Purpose: To describe a new surgical approach to the anterior thoracic spine.

Study Desing/Setting: Literature overview and case report utilizing the lateral approach to access the anterior thoracic spine.

Methods: The lateral access surgery was first developed to approach the lumbar spine. Here we present our experience using this technique to access the thoracic spine in a minimally invasive fashion.

Results: This minimally disruptive spine procedure allowed an adequate approach to the thoracic discs, with less soft tissue aggression and intra-operative bleeding, shorter operation time and hospital stay, lower postoperative pain and earlier return to work. Therefore, the potential complications with an anterior open approach were avoided, and the procedures were performed through a single, 5-6 cm incision.

Conclusion: The modification of the XLIF technique for thoracic spine is a safe and effective procedure to achieve fusion and indirect decompress the neural structures. This minimal invasive procedure allows through a single small incision the treatment of different conditions of the thoracic spine without the need of posterior supplementation and huge incisions, avoiding open approach complications and maximizing results due to less tissue trauma and adequate exposure of the thoracic discs.

Keywords: Spine; Lateral access; Thoracic spine; Minimally invasive

\section{Introduction}

The thoracic spine accounts for less than $1 \%$ of all clinically relevant disc protrusion [1,2] and are estimated to occur in 1 per $1,000,000$ patients per year [3]. A vague medical history and the relative rarity of the condition often results in delayed diagnosis. Because of improvements in diagnostic imaging, the evaluation of thoracic disc herniations getting more frequent [4,5]. However, it is imperative, therefore, to understand the presentation, evaluation, and alternatives for the treatment of this disease. The thoracic spine disorders have undergone dramatic changes in neurosurgical management, diagnosis and treatment. The standard procedure for thoracic spine conditions, such as degenerative diseases, instability or disc herniation is fusion [5]. For many authors, the current way to access the thoracic spine is the transthoracic approach3. This technique allows a wide direct approach to the anterior aspect of the thecal sac, which is very important when dealing with central disc herniation. It enables adherent disc material or adherent posterior longitudinal ligament (PLL) to be carefully dissected away from the dura; the ability to preserve spinal stability, and to perform interbody fusion when indicated. Complications or consequences related to this approach include: pneumothorax, hemothorax, chylothorax, postthoracotomy pai, duropleural fistula with cerebrospinal fluid effusion, pneumonia, flail musculature, great vessel injury, and sympathectomy [6].

The evolution of surgical techniques has been increased due to the development of minimally invasive approach to the thoracic spine. The video-assisted thoracic surgery (VATS) had been first reported in 1993 for spinal disease by Mack et al. [7]. VATS allows a significantly reduction in chest wall morbidity related to the tradicional thoracotomy [8]. These included a reduction of the postoperative incisional pain and intercostals neuralgia. This technique provides a greater access to more vertebral levels through smaller incisions, when compared to transthoracic approach, but still presents some complications, such as intercostal neuralgia (7.7\%), symptomatic atelectasis $(6.4 \%)$, excessive (>2000cc) intraoperative blood loss (2.5-5.5\%), pneumonia (1-3\%), wound infections (1-3\%), chylothorax (1\%), tension pneumothorax, long thoracic nerve injury and pulmonary embolism [9].

To avoid these complications, the eXtreme Lateral Interbody Fusion (NuVasive, Inc., San Diego, Ca) was modified to access the thoracic spine, using the same dilator system (Maxcess System, NuVasive Inc., San Diego, Ca), with all surgical and clinical advantages already described for lumbar spine[10].

This current report describes the modification of the XLIF technique for thoracic spine surgery. This minimally disruptive spine procedure allows an adequate approach to the thoracic discs, with less soft tissue aggression and shorter hospital stay, with lower postoperative pain and earlier return to work. Because the procedure is performed through a small incision and without the need of pedicle screws supplementation, the operative time and blood loss are much reduced. By an implantation of a larger implant that rests on both sides

*Corresponding author: Leonardo Oliveira, Institute of Spine Pathology (IPC), Brazil, Tel: (11) 2936.8838; Email: leonardo@patologiadacoluna.com.br

Received July 08, 2015; Accepted July 29, 2015; Published July 31, 2015

Citation: Pimenta L, Marchi L, Fortti F, Oliveira L, Amaral R, et al. (2015) Minimally Invasive Lateral Approach to the Thoracic Spine - Case Report and Literature Overview. J Spine 4: 240.doi:10.4172/21657939.1000240

Copyright: $\odot 2015$ Pimenta L, et al. This is an open-access article distributed under the terms of the Creative Commons Attribution License, which permits unrestricted use, distribution, and reproduction in any medium, provided the original author and source are credited. 
of the ring apophysis, the XLIF technique maximizes the endplate support and allows a standalone construction with a low incidence of severe cage subsidence. Therefore, the potential complications with an anterior open approach can be avoided, and the procedure can be done through a single, 5-6 $\mathrm{cm}$ incision.

\section{Material and Methods}

\section{Patient selection and surgical indications}

Patients who present with axial thoracic back pain with or without severe central canal stenosis or disc herniations were considered candidates for this surgery. The treatment levels range from T4-5 to T11-12. Contraindications included significant rotatory scoliosis. The group of patients is essentially the same as those with degenerative disc disease and considered candidates for fusion. A preoperative MRI should be examined to identify the position of the aorta. In the midthoracic spine, especially in scoliotic patients, the aorta tends to lie at the left lateral aspect of the vertebral body [11]. In this case, some surgeons will approach from the opposite side and ensure that their disc preparation instruments do not pass more than $2 \mathrm{~mm}$ through the contralateral annulus.

\section{Surgical technique}

The patient is placed on a bendable surgical table in a direct lateral decubitus $\left(90^{\circ}\right)$ position. If the surgery involves lumbar and thoracic levels, the patient is positioned so the table break is directly under the greater trochanter. If the surgery includes only thoracic levels, the patient is positioned with the table break under the mid-surgical level. The patient is then secured with tape at the following locations (Figure 1). In scoliotic cases, is preferable to work on the convexity of the curve. In degenerative cases, is preferable to use the left side, working on the arterial side.

Once the patient is taped, the fluoroscopy is utilized to confirm targeted disc space location and adjust the table to provide true AP and
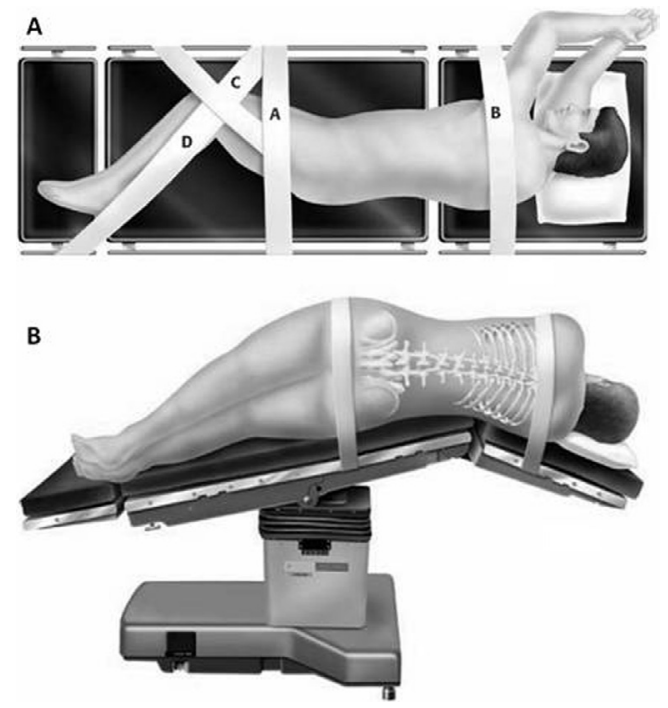

Figure 1: A. Patient Positioning: A) Tape just below the iliac crest; B) Tape over the thoracic region (ensuring tape does not interfere with the surgical exposure of the level of interest); C) Tape from the iliac crest to the knee, then secured to the table; D) Tape from one side of the table to the knee, past the ankle, then secured to the other side of the table. B. Surgical Table. The table should be break at the targeted level to increase the distance between the ribs and facilitate the access to the disc space.
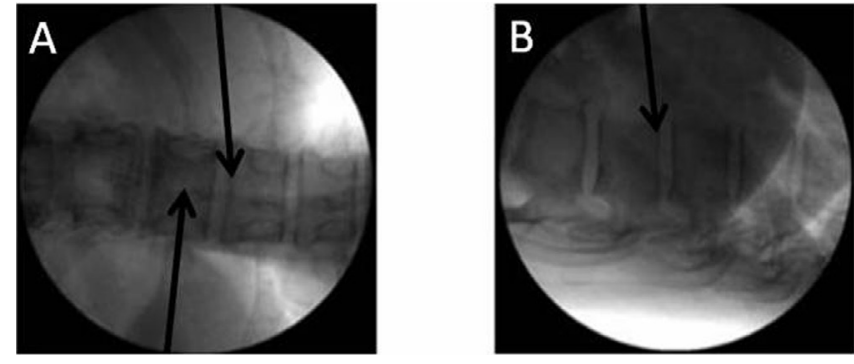

Figure 2: Proper disc Location. A) Spinous process centered between pedicles and distinct endplates (black arrows). B) Lateral view showing distinct endplates (black arrow).

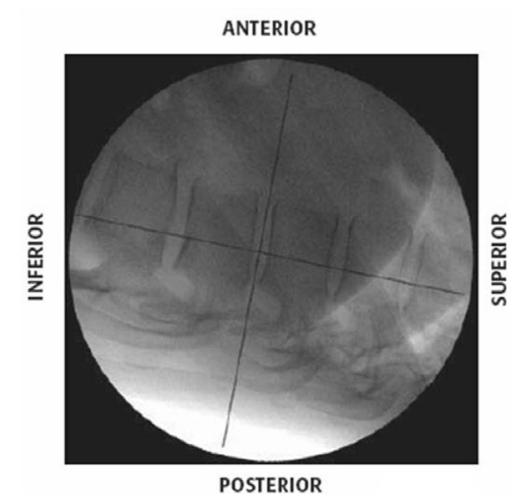

Figure 3: Disc space location in order to define the correct position of the skin incision.
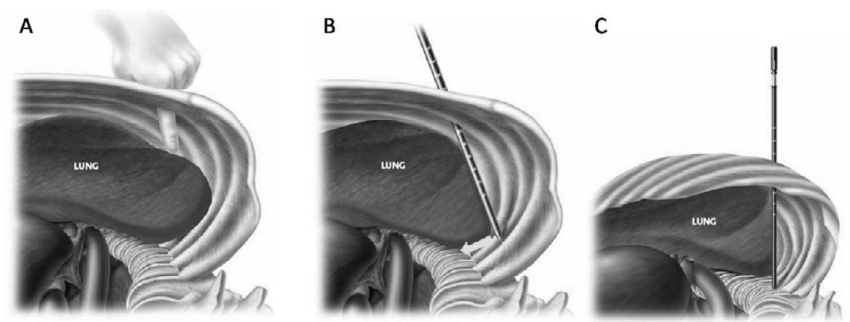

Figure 4: A) Index finger palpating and displacing the thoracic cavity structures B) Introduction of the first dilator. Note the posterior direction of the dilator C) Slide down the dilator to access the intersection of the rib head and the vertebral body.

lateral images when the C-Arm is horizontal or vertical, respectively (Figure 2). The table should be independently adjusted for every approached level in order to maintain this relationship. A K-wire is placed perpendicular to the spine at the index level. The other K-Wire is placed parallel to the spine at the posterior middle-third of the disc space or vertebra (Figure 3). Two marks will be made to define the location of the skin incision.

\section{Skin incision and surgical approach}

The incision will be made parallel to the ribs at the intersection of the skin markings. The surgeon will be able to access a single level by passing between the ribs. For expanded access, utilized in multilevel cases, it will be necessary to dissect a small section of the rib head. Care should be taken to preserve the neurovascular bundle that lies under the inferior aspect of each rib. Dissection will be performed through the subcutaneous tissue down to the ribs or intercostal space (Figure 4). 
Citation: Pimenta L, Marchi L, Fortti F, Oliveira L, Amaral R, et al. (2015) Minimally Invasive Lateral Approach to the Thoracic Spine - Case Report and Literature Overview. J Spine 4: 240.doi:10.4172/21657939.1000240

\section{Thoracic access}

Following the parietal pleura insertion, the index finger is used to enter the pleural space, palpating the lung and displacing anteriorly the pleural structures. The initial dilator will be introduced posteriorly into the thoracic cavity sliding down to the intersection of the rib head and the vertebral body. There is no need to deflate any of the lungs.

Once the initial Dilator is positioned, fluoroscopic image should be taken to confirm position. A lateral image will confirm that the dilator is positioned in the posterior third of the disc space and parallel to the disc, and a cross-table AP image should confirm that the dilator is docked onto, and in the plane of, the disc space (Figure 5). The next two dilators will be subsequently passed over the initial dilator down to access the disc space. The utilized dilators have 6,9 and $12 \mathrm{~mm}$, respectively.

\section{Exposure}

The working portal is introduced over the third Dilator. The utilized blades range from $50 \mathrm{~mm}$ to $150 \mathrm{~mm}$, varying $10 \mathrm{~mm}$ from each one and totalizing 11 different sizes. A cross-table AP fluoroscopy is used to confirm the correct positioning. The confirmation is essential to ensure that the Blades are parallel with the disc space (Figure 6). Under direct visualization, shims can be used to effectively widen or lengthen the access driver blades to retract the lung if necessary in order to gain access to the disc space.

\section{Discectomy and disc space preparation}

The parietal pleura is incised in order to gain access to the disc space. Under direct visualization a thorough discectomy is performed using standard instruments. The posterior annulus can be left intact, with the annulotomy window centered in the anterior half of the disc space and wide enough to accommodate a large implant, which will provide indirect decompression of the spinal cord by ligamentotaxis.
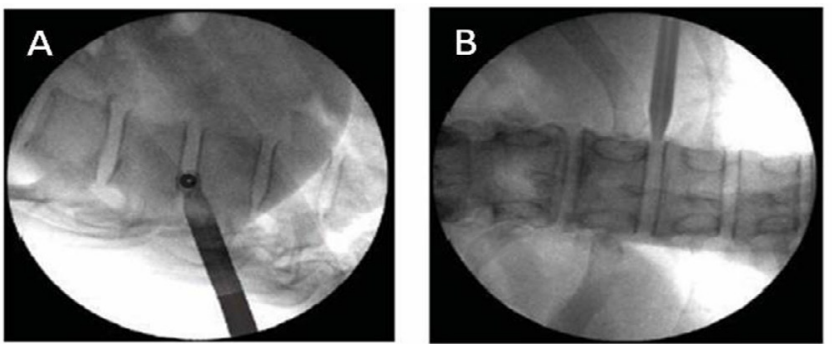

Figure 5: A) Lateral view showing the positioning of the initial dilator in the posterior third of the disc space and parallel to the disc. B) Cross-table AP view showing the perfect position of the dilator, docked onto the spine and aligned to the disc space.

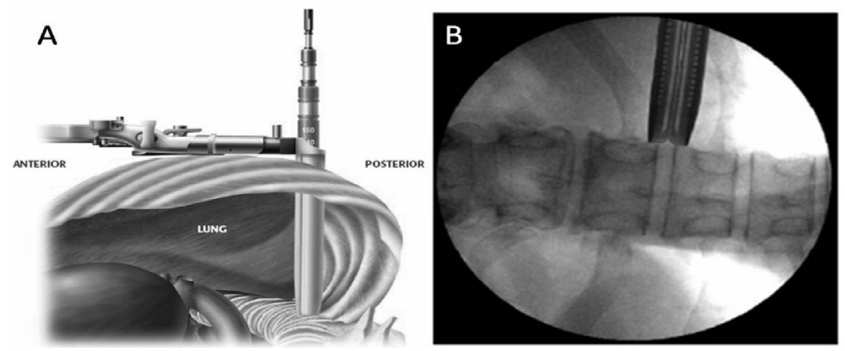

Figure 6: A) The access driver is inserted over the dilators and will be the working portal. B) Fluoroscopy to confirm the exact positioning of the access driver.

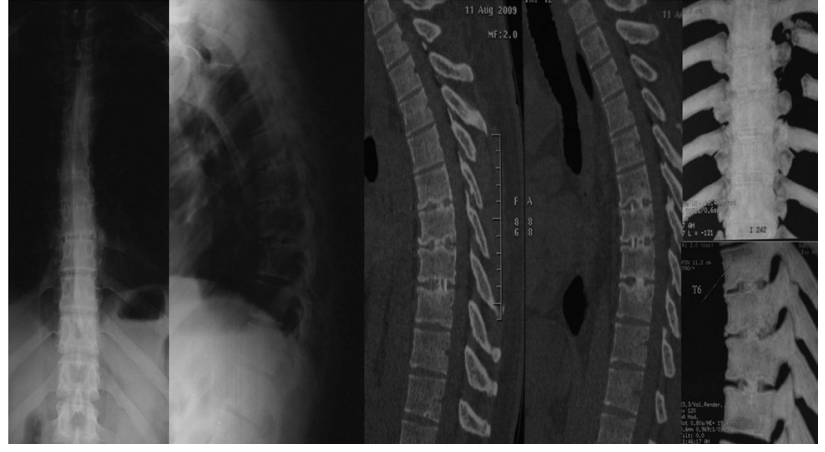

Figure 7: X-rays and CT scans showing solid fusion two years after the thoracic XLIF procedure.

Care should be taken near the contralateral annulus to avoid damaging structures deep to the annulus, as indicated on the preoperative MRI. Disc removal and release of the contralateral annulus with a Cobb Elevator provides the opportunity to place a long implant that will rest on both lateral margins of the apophyseal ring, maximizing endplate support and providing strong support for disc height restoration. The use Transcranial Motor Evoked Potential (MEP) to monitor spinal cord health and integrity during surgery is mandatory. After standard annulotomy and disc space preparation, a subsequent MEP reading can be taken to ensure no compromise of the motor pathways of the spinal cord.

\section{Implant sizing and placement}

Different sizers may be used to distract the disc space and measure the appropriately trial. The AP fluoroscopy is essential to guide the trial placement into the disc space. The distal end of the trial needs to reach the contra lateral margin of the disc space. Proper position is verified using lateral fluoroscopy. Larger trials will be used until desired fit and placement is achieved. When the implant is selected, it will be filled with graft material and attached to the inserter. The implant is then impacted into the disc space, being always monitored by AP fluoroscopy.

\section{Closure}

Once the procedure is completed, the working portal is gently removed while verifying the absence of significant bleeding. A chest tube may is routinely used, but when parietal pleura is preserved, the chest tube can be avoided. be placed and theThe wound must be closed in a layered fashion. The skin is closed using standard subcuticular suture.

\section{Case Examples}

\section{Case 1: Thoracic access}

ACVCA, 49 years old, presented with thoracic and left leg pain for more than one year. The magnetic resonance image showed disc herniation and degenerative disc disease at T6-T7, T7-T8 and T8-T9. The lateral procedure was performed without any intraoperative intercurrence in 110 minutes with less than $50 \mathrm{cc}$ of blood loss. Approximately $3-4 \mathrm{~cm}$ of the T6 and T7 ribs were resected to achieve additional exposure. Patient was discharged 48 hours after the procedure. The Visual Analogue Scale showed improvement of $80 \%$ six weeks after surgery and was maintained during all follow up visits. Two years radiological images showed solid fusion at all operated levels (Figure 7). 


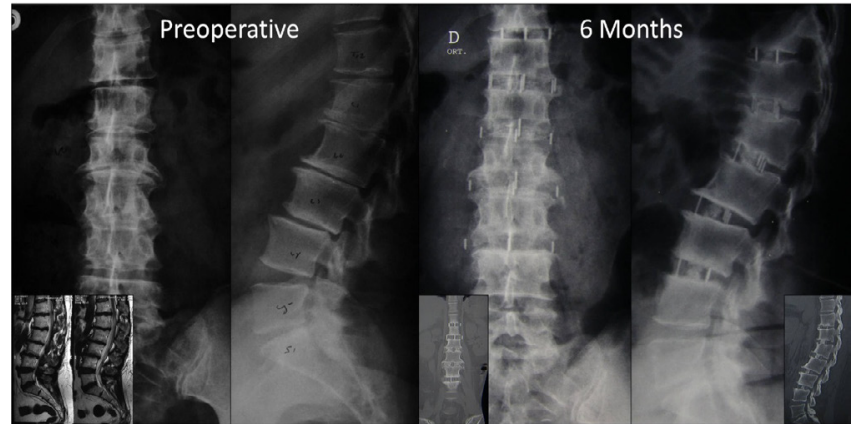

Figure 8: Preoperative images showing incipient osteophytes, degenerative disc disease and retrolisthesis between T11-T12 to L3-L4. Six months after the procedure, A-P and lateral x-rays show good positioning of the cages, absence of important subsidence and good sagittal and coronal balance. CT scan shows initial bone formation at all operated levels.

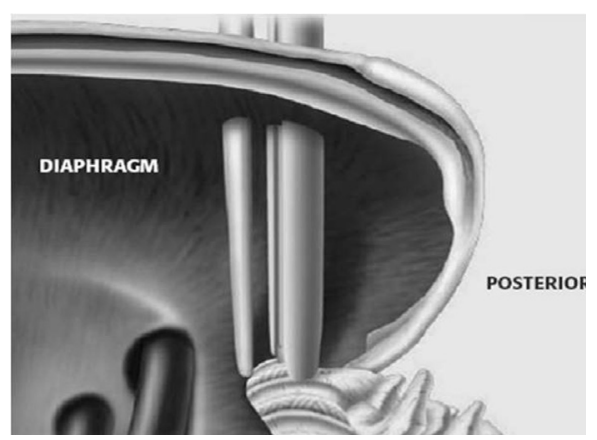

Figure 9: Transitional level access by XLIF approach. The retractor must pass through the diaphragm to address the T12-L1 disc space.

\section{Case 2: Thoracolumbar access}

FFM, 55 years old, presented with back pain for more than five years. The conservative treatment didn't show any satisfactory results. Preoperative resonance images showed stenosis and degenerative disc disease from T11 to L3-L4. The lateral procedure was performed in 130 min without intra operative complications. The blood loss was less than 50cc. All levels were accessed by two small incisions. Patient deambulated 4 hours after the procedure and was discharged less than 24 hours after surgery. Six months after surgery, CT scan shows bone bridging at all operated levels (Figure 8).

\section{Transitional level (T12-L1) considerations}

To access the T12-L1 level, the access driver will pass between the ribs, through the diaphragmatic attachment at the ribs, and into the retroperitoneal space. This approach is important when lumbar levels are also being addressed (Figure 9).

\section{Discussion}

The XLIF technique is a modification of the retroperitoneal approach to the lumbar spine, firstly presented by Luiz Pimenta in 2001, which performed more than 100 lateral trans-psoas surgeries performed between 1998 and 2001 [12]. In comparison to other lumbar techniques, the lateral approach has several advantages. First, there is no necessity of a general surgeon to perform the access because approaching laterally the manipulation of the peritoneum and great vessels is eliminated. Second, due to direct visualization of the tissues, all dissection occurs without impairment of depth perception, what happens in laparoscopic approaches to the lumbar spine [13-15]. Third, the far lateral approach avoids disturbance of the superior hypogastric nerve plexus and the incidence of retrograde ejaculation in XLIF procedure is null. Because the procedure is performed through a small incision and without the need of pedicle screws supplementation, the operative time and blood loss are much reduced. By an implantation of a larger implant that rests on both sides of the ring apophysis, the XLIF technique maximizes the endplate support and allows a standalone construction with a low incidence of severe cage subsidence. Restoring the disc height and providing stability by preserving the constraining ligaments, it is also possible to indirect decompress the neural structures and correct the coronal, sagittal and spinopelvic balance.

Access for the thoracic spine has a lot of possible risks [16-18]. The modification of the XLIF technique for thoracic spine is a safe and effective procedure to achieve fusion and indirect decompress the neural structures. This minimal invasive procedure allows through a single small incision the treatment of different conditions of the thoracic spine without the need of posterior supplementation and huge incisions, avoiding open approach complications and maximizing results due to less tissue trauma and adequate exposure of the thoracic discs, as shown in an initial multicenter clinical experience for the treatment of symptomatic disc herniation [19]. In conclusion, this approach provides simple exposure of difficult access pathology, avoiding many of the complications inherent to open traditional thoracic exposures.

\section{References}

1. Burke TG, Caputy AJ (2000) Treatment of thoracic disc herniation: evolution toward the minimally invasive thoracoscopic technique. Neurosurg Focus. 9: e9.

2. Rogers MA, Crockard HA (1994) Surgical treatment of the symptomatic herniated thoracic disk. Clin Orthop 300: 70-78.

3. Vollmer DG, Simmons NE (2000) Transthoracic approaches to thoracic disc herniations. Neurosurg Focus 9: e8.

4. Snyder LA, Smith ZA, Dahdaleh NS, Fessler RG (2014) Minimally invasive treatment of thoracic disc herniations. Neurosurg Clin N Am 25: 271-277.

5. Yoshihara $\mathrm{H}$ (2014) Surgical treatment for thoracic disc herniation: an update. Spine 39: E406-412.

6. McCormick WE, Will SF, Benzel EC (2000) Surgery for thoracic disc disease. Complication avoidance: overview and management. Neurosurg Focus 9: e13.

7. Mack MJ, Regan JJ, Bobechko WP, Acuff TE (1993) Application of thoracoscopy for diseases of the spine. Ann Thorac Surg 56: 736-738.

8. Newton PO, Marks M, Faro F, Betz R, Clements D, et al. (2003) Use of videoassisted thoracoscopic surgery to reduce perioperative morbidity in scoliosis surgery. Spine 28: S249-254.

9. McAfee PC, Regan JR, Zdeblick T, Zuckerman J, Picetti GD, et al. (1995) The incidence of complications in endoscopic anterior thoracolumbar spinal reconstructive surgery. A prospective multicenter study comprising the first 100 consecutive cases. Spine 20: 1624-1632.

10. Ozgur BM, Aryan HE, Pimenta L, Taylor WR (2006) Extreme Lateral Interbody Fusion (XLIF): a novel surgical technique for anterior lumbar interbody fusion. Spine J 6: 435-443.

11. Qiu XS, Jiang H, Qian BP, Wang WJ, Zhu F, et al. (2014) Influence of prone positioning on potential risk of aorta injury from pedicle screw misplacement in adolescent idiopathic scoliosis patients. J Spinal Disord Tech 27: E162-E167.

12. Pimenta $L$ (2001) Lateral endoscopic transpsoas retroperitoneal approach for lumbar spine surgery.

13. Brau SA (2002) Mini-open approach to the spine for anterior lumbar interbody fusion: description of the procedure, results and complications. Spine $\mathrm{J}$ Off $\mathrm{J}$ North Am Spine Soc 2: 216-223.

14. Garg J, Woo K, Hirsch J, Bruffey JD, Dilley RB (2010) Vascular complications of exposure for anterior lumbar interbody fusion. J Vasc Surg 51: 946-950.

15. Rajaraman V, Vingan R, Roth P, Heary RF, Conklin L, et al. (1999) Viscera and vascular complications resulting from anterior lumbar interbody fusion. $J$ Neurosurg 91: 60-64 
Citation: Pimenta L, Marchi L, Fortti F, Oliveira L, Amaral R, et al. (2015) Minimally Invasive Lateral Approach to the Thoracic Spine - Case Report and Literature Overview. J Spine 4: 240.doi:10.4172/21657939.1000240

16. Pettiford BL, Schuchert MJ, Jeyabalan G, Landreneau JR, Kilic A, et al (2008) Technical challenges and utility of anterior exposure for thoracic spine pathology. Ann Thorac Surg 86:1762-1768.

17. Fessler RG, Sturgill M (1998) Review: complications of surgery for thoracic disc disease. Surg Neurol 49: 609-618.
18. Ikard RW (2006) Methods and complications of anterior exposure of the thoracic and lumbar spine. Arch Surg Chic 1960 141: 1025-1034.

19. Uribe J, Smith W, Pimenta L, Härtl R, Dakwar E, et al. (2012) Minimally invasive lateral approach for symptomatic thoracic disc herniation: Initial multi-center clinical experience. J Neurosurg Spine 16: 264-279. 